O Open Access Full Text Article

\title{
Retrospective analysis of an intraoperative
} aberrometry database: a study investigating absolute prediction in eyes implanted with low cylinder power toric intraocular lenses

This article was published in the following Dove Press journal:

Clinical Ophthalmology

\author{
Robert J Cionni' \\ Michael Breen ${ }^{2}$ \\ Cody Hamilton ${ }^{3}$ \\ Ravaughn Williams ${ }^{4}$ \\ 'The Eye Institute of Utah, Salt Lake City, \\ UT, USA; ${ }^{2}$ North America Clinical \\ Development and Medical Affairs, Alcon \\ Laboratories, Inc., Fort Worth, TX, USA; \\ ${ }^{3}$ Biostatistics, Alcon Laboratories, Inc., \\ Fort Worth, TX, USA; ${ }^{4}$ Global Clinical \\ Development and Medical Affairs, Alcon \\ Laboratories, Fort Worth, TX, USA
}

Purpose: To evaluate the spherical equivalent outcomes of intraoperative aberrometry (IA) power calculations compared with the surgeons' preoperative power calculations in eyes implanted with AcrySof ${ }^{\mathbb{B}}$ IQ T3 intraocular lenses (IOLs).

Patients and methods: We assessed data collected by an IA system from multiple centers in the United States. Data was from patients who had undergone cataract extraction by phacoemulsification with the use of the Optiwave Refractive Analysis System and whose eyes had been implanted with model SN6AT3 (1.5 diopter [D] at IOL plane) aspheric lenses. The analysis was performed in 2 stages: hypothesis generation and confirmatory testing. Confirmatory endpoints were a comparison of absolute prediction errors for IA for the implanted IOL versus preoperative formula power calculations.

Design: Retrospective analysis of preoperative, intraoperative, and postoperative data concerning eyes implanted with model SN6AT3 (1.5 D) aspheric lenses from the AnalyzOR ${ }^{\text {TM }}$ database.

Results: Mean absolute IA prediction error was significantly lower than preoperative prediction error (paired difference: $-0.06 \mathrm{D} ; p<0.0001$ ); this was mirrored by the median paired difference of $-0.04 \mathrm{D}(p<0.0001)$. For eyes where the power of the implanted IOL differed from the power of the preoperatively planned lens, mean and median paired differences in prediction errors were greater: $-0.13 \mathrm{D}(p<0.0001)$ and $-0.15 \mathrm{D}(p<0.0001)$, respectively. The percentage of eyes with prediction error $\leq 0.50 \mathrm{D}$ was significantly higher with IA $(83.4 \%, n=5388 / 6460)$ than with the preoperative formula $(76.5 \%, n=4942 / 6460$, $p<0.0001)$. When the powers of the implanted IOL and the preoperatively planned lens were different, the percentage of eyes with prediction error $\leq 0.50 \mathrm{D}$ was $83.3 \%(2155 / 2587)$ for IA and $68.8 \%(1781 / 2587, p<0.0001)$ for the preoperative formula.

Conclusion: IA produces more accurate spherical equivalent outcomes for eyes implanted with a low toric IOL than the preoperative formulas.

Keywords: astigmatism, intraocular lenses, low toric lenses, spherical equivalent outcomes

\section{Introduction}

A variety of diagnostic biometric devices and formulas are available for preoperative calculation of the power of toric intraocular lenses (IOL) to correct astigmatism during cataract surgery. ${ }^{1,2}$ After making these calculations, surgeons have a choice during the surgery itself. They may choose to use the IOL power based on their original preoperative plan, use the IOL power recommended by intraoperative
The Eye Institute of Utah, 755 E 3900 S,

Salt Lake City, UT 84107, USA

Email rcionni@theeyeinstitute.com 
aberrometry (IA), or use an IOL power in between the preoperatively planned and IA-recommended powers. Although empirical formulas assist IOL power calculations, they are based on the assumption that all clinical settings and surgeons operate in the same way. However, this is not accurate. For example, the technique used to measure axial length and surgical technique varies across settings and among surgeons. ${ }^{1}$ Therefore, to improve refractive outcome, the surgeon should optimize the surgeon factor (lens constant, defined as the theoretical difference between the corneal height and the effective optical plane of the IOL) in the IOL power calculation.

However, if the toric IOL is misaligned or rotates postoperatively, its effect on correcting astigmatism at the planned axis of alignment diminishes, and the IOL may induce increased astigmatism at another axis. ${ }^{3}$ Surgically induced astigmatism caused by the primary incision might also render the preoperative estimation less accurate. ${ }^{4}$ However, if the residual astigmatism is $\leq 0.50 \mathrm{D}$ but the surgeon residual SE error is $>0.50 \mathrm{D}$ from the target, the patient will likely be unhappy with the refractive result. Therefore, the surgeon hopes to attain both the desired spherical equivalent and cylinder outcomes.

The Optiwave Refractive Analysis System ${ }^{\circledR}$ (Alcon Laboratories, Inc.; Fort Worth, TX, USA) is an IA system for surgeons implanting IOLs. ${ }^{4}$ The Optiwave Refractive Analysis System provides real-time measurements of the eye during surgery, after the crystalline lens has been removed and the anterior chamber refilled with either balanced salt solution or a cohesive ophthalmic viscosurgical device. This system facilitates toric IOL spherical and cylinder power calculations, and accounts for surgically induced astigmatism and the contribution of the posterior cornea in its aphakic refraction measurement. The system's database, AnalyzOR ${ }^{\mathrm{TM}}$, securely stores patients' preoperative, intraoperative, and postoperative data and uses this information to optimize the lens constants and regression coefficients used in the IA IOL power formula. ${ }^{4}$ Compared with conventional preoperative measurement-based IOL power calculation methodologies, IA demonstrated significantly improved accuracy $(p<0.0001)$ in predicting IOL power in 215 patients undergoing cataract surgery with a history of myopic LASIK or photoreactive keratectomy. ${ }^{4}$ In another study of 124 eyes, IA was associated with reduced refractive astigmatism. ${ }^{5}$ Other studies have reported no significant difference between preoperative measurement and IA for selection of intraocular lens sphere power; however, sample sizes in these studies were comparatively small (51 to 160 eyes). ${ }^{6-8}$
The objective of this study was to evaluate refractive outcomes of the IA power calculation compared with the surgeon's preoperative power calculation in eyes implanted with AcrySof IQ T3 IOLs, ${ }^{9}$ using data from a large database of patients who have undergone cataract surgery. The primary endpoints were the following: 1) mean and median differences between the absolute IA prediction error for the implanted IOL and back calculated preoperative formula absolute prediction error, and 2) difference in the percentage of eyes with IA absolute prediction error $\leq 0.50 \mathrm{D}$ for the implanted $\mathrm{IOL}$ and the percentage of eyes with back calculated preoperative formula absolute prediction error $\leq 0.50 \mathrm{D}$. These endpoints were determined for the overall sample (all eyes) and for eyes where the power of the implanted IOL implanted was different from the power of the preoperatively predicted lens.

\section{Materials and methods}

\section{Study design}

This study was a retrospective analysis of data collected by the IA system from multiple centers in the United States. The Salus Independent Review Board, Austin, TX, approved this study and the requested Institutional Review Board/Independent Ethics Committee Waiver of Informed Consent before the first database transfer. Data were collected only from sites in the United States that granted the waiver. With the exception of obtaining informed consent, these analyses were conducted in accordance with the principles of the Declaration of Helsinki, and in compliance with Good Clinical Practice, the US Food and Drug Administration 21 CFR 812, and all other applicable regulations.

\section{Statistical methods}

Data were from the AnalyzOR database, which collects preoperative, intraoperative, and postoperative data from cataract refractive surgery cases. The present analysis was limited to AnalyzOR data from patients who had undergone cataract extraction by phacoemulsification in at least 1 eye with the use of the Optiwave Refractive Analysis System; had preoperative, intraoperative, and postoperative data in the AnalyzOR database; and whose eyes had been implanted with SN6AT3 (1.5 D at IOL plane) aspheric lenses (Alcon Laboratories, Inc.; Fort Worth, TX, USA). Data for patients who met these criteria for analysis were anonymized and transferred to Alcon. Data were 
analyzed in 2 stages: hypotheses were generated using a random $5 \%$ of the sample (exploratory stage), then these hypotheses were tested using the remaining $95 \%$ of the sample (confirmatory stage). The number of eyes in the confirmation dataset provided an adequate effective size for the degrees of freedom required by the analyses, based on statistical guidelines. ${ }^{10}$ These analyses examined IA absolute prediction error and preoperative absolute prediction error.

IA absolute prediction error for an implanted IOL is the absolute value of the difference between the manifest subjective refraction spherical equivalent (MRSE) predicted intraoperatively for that IOL and the postoperative MRSE achieved with that IOL. The preoperative absolute prediction error is the absolute value of the difference between the MRSE predicted by the IOL formula used for the preoperatively planned lens and the MRSE that would have been achieved if the preoperatively planned IOL had been implanted. ${ }^{11}$

The study endpoints were summarized with statistics appropriate to the scale (continuous or categorical). All continuous outcomes were analyzed as paired differences between IA prediction error and the preoperative prediction error; this accounted for the correlation between the two errors recorded per eye (one for IA and the other for the preoperative formula). The analysis of the categorical outcomes was based on a chi-square statistic accounting for this correlation as well. The summary statistics were also stratified by preoperative formula for the three most common formulas (Holladay 1, Holladay 2, and SRK/T). A separate sensitivity analysis using those patients with only one eye in the database was performed to determine if there was any impact of including patients with two eyes in the database (these eyes would be correlated).

\section{Study endpoints}

Six primary endpoints were selected for confirmatory testing: 1) mean difference between the IA absolute prediction error for the implanted IOL and the preoperative absolute prediction error back calculated for the preoperative formula; 2) median difference between the IA absolute prediction error for the implanted IOL and the preoperative absolute prediction error back calculated for the preoperative formula; 3 ) mean difference between the IA absolute prediction error for the implanted IOL and the preoperative absolute prediction error back calculated for the preoperative formula, in eyes where the power of the implanted IOL was different from the power of the preoperatively planned lens; 4) median difference between the IA absolute prediction error for the implanted IOL and the preoperative absolute prediction error back calculated for the preoperative formula, in eyes where the power of the implanted IOL was different from the power of the preoperatively planned lens; 5) difference in the percentages of eyes with IA absolute prediction error $\leq 0.50 \mathrm{D}$ for the implanted IOL and eyes with preoperative absolute prediction error back calculated for the preoperative formula $\leq 0.50 \mathrm{D}$; and 6) difference in the percentages of eyes with IA absolute prediction error $\leq 0.50 \mathrm{D}$ for the implanted IOL and eyes with preoperative absolute prediction error back calculated for the preoperative formula $\leq 0.50 \mathrm{D}$, and where the power of the implanted IOL was different from the power of the preoperatively planned lens.

Exploratory endpoints in this study included: 1) percentages of eyes where the IA-recommended lens power differed from the preoperatively planned lens power and the surgeon implanted the IA-recommended lens power, the preoperatively planned lens power, or another lens power; 2) percentages of eyes where the power of the IA-recommended lens and the power of the preoperatively planned lens were the same and the surgeon implanted the preoperatively planned and IA-recommended lens power or another lens power.

\section{Results}

The anonymized data set that met the predefined inclusion criteria contained 6800 eyes. Of these, 340 were used in the hypothesis-generating phase of the analyses. A total of 6460 eyes from 5761 patients were included in the confirmatory phase of the analysis, with refractive results collected on average 43 days postoperatively. All patients had undergone unilateral or bilateral implantations, and the mean $( \pm \mathrm{SD})$ preoperative astigmatism was $1.22( \pm 0.44) \mathrm{D}$ (range, 0 to $5.32 \mathrm{D}$ ). A variety of formulas were used to calculate preoperative lens power (Table 1). Results obtained using the three most commonly used formulas, Holladay 1 and 2 and SRK/T, were comparable (Table 2).

\section{Primary outcomes}

The mean paired difference between the IA absolute prediction error and the preoperative absolute prediction error was $-0.06 \mathrm{D}(p<0.0001)$; the median paired difference was $-0.04 \mathrm{D}(p<0.0001)$ (Table 3$)$. In eyes where the power of the implanted IOL differed from the power of the preoperatively planned lens, the mean and median paired differences in absolute prediction errors were greater: -0.13 
Table I Preoperative lens power formulas used $(\mathrm{N}=6460)$

\begin{tabular}{|l|l|l|}
\hline Formula & $\mathbf{n}$ & Percent \\
\hline Haigis & 147 & 2.3 \\
Hoffer Q & 171 & 2.7 \\
Holladay I & 1038 & 16.1 \\
Holladay 2 & 3077 & 47.6 \\
Olsen & 3 & $<0.01$ \\
Other & 228 & 3.5 \\
SRK/T & 1796 & 27.8 \\
\hline
\end{tabular}

D $(p<0.0001)$ and $-0.15 \mathrm{D}(p<0.0001)$, respectively (Table 3).

The percentage of eyes with an absolute prediction error $\leq 0.50 \mathrm{D}$ was significantly higher with IA (83.4\%) compared with the preoperative formula $(76.5 \%$, $p<0.0001$ ), resulting in a difference of $6.9 \%$ (Table 3 ). In addition, the percentage of eyes with an absolute prediction error $\leq 0.50 \mathrm{D}$ and where the power of the implanted IOL was different from that of the preoperatively planned lens was significantly higher with IA (83.3\%) than with the preoperative formula $(68.8 \%, p<0.0001)$, resulting in a difference of $14.5 \%$ (Table 3). A sensitivity analysis of the 5062 patients with only one eye in the database yielded results consistent with the combined cohort (Table 3).

The IA-recommended lens spherical power differed from the preoperatively planned lens spherical power for 3627 eyes (Figure 1). For these eyes, surgeons were more likely to use the IA-recommended lens power $(47.3 \%$ of eyes) than to use the preoperatively planned lens power $(37.7 \%)$ or another approach (15.0\%) (Figure 1).

\section{Discussion}

The predictability of the post-operative spherical equivalent (SE) is vitally important to patients electing to undergo astigmatism reduction using a toric IOL at the time of cataract surgery. If the surgeon achieves a result of less than $0.5 \mathrm{D}$ of cylinder yet has greater than $0.5 \mathrm{D}$ of SE error, the patient will likely be dissatisfied with the result and may require glasses. This study of more than 6000 SN6AT3 IOL implantations is the first large analysis of absolute prediction errors of IA compared with conventional biometry in patients receiving toric IOLs to treat low amounts of astigmatism. The results showed that the IA mean and median absolute prediction errors were statistically significantly lower than the preoperatively planned IOL absolute prediction errors ( $p<0.0001$ for both paired differences). This difference was even greater in eyes in which the spherical power of the

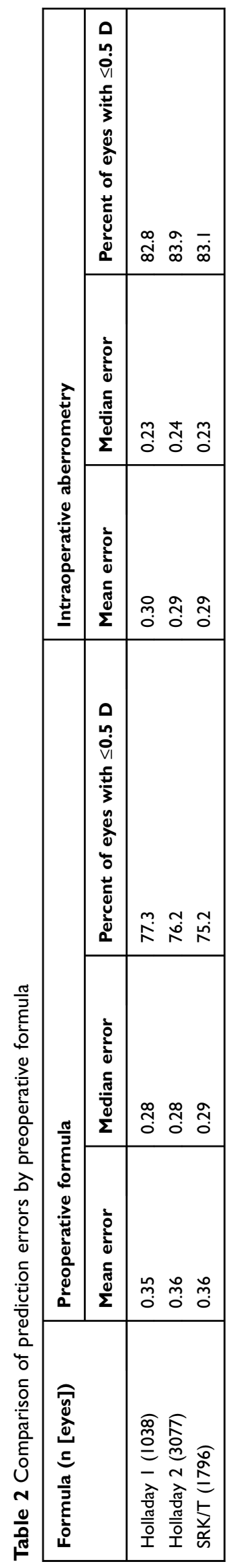




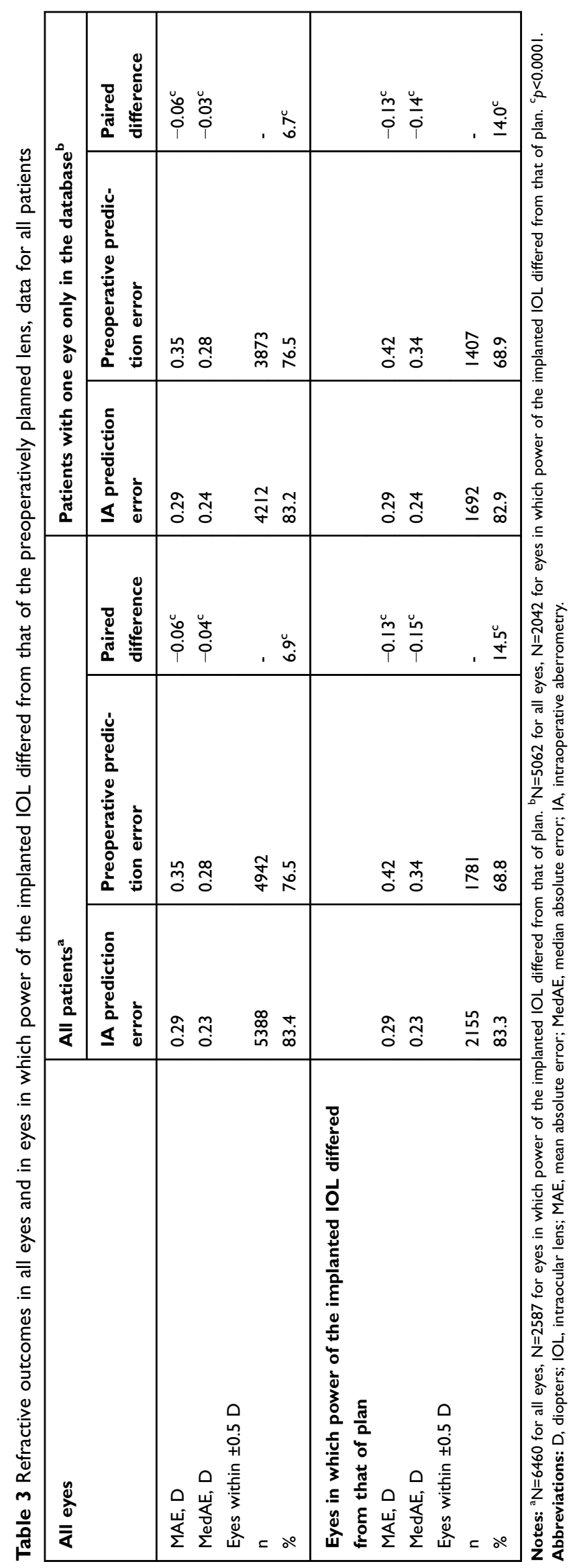




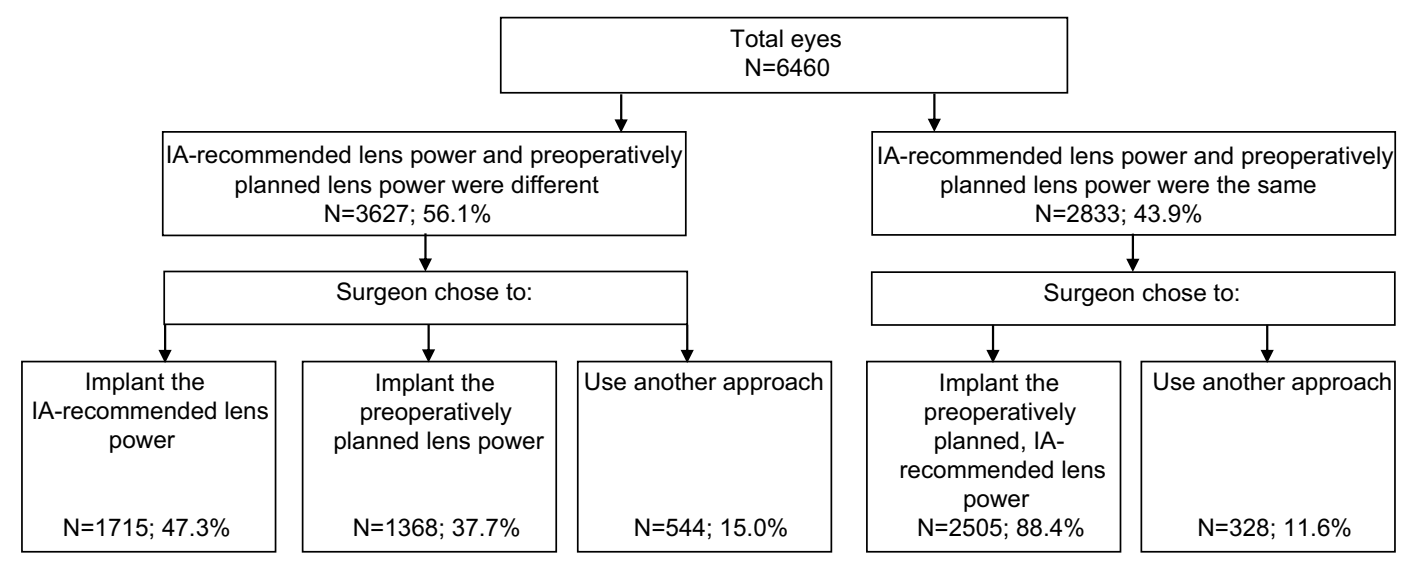

Figure I Lens power implanted.

Abbreviation: IA, intraoperative aberrometry.

SN6AT3 IOL was different from the spherical power of the preoperatively planned lens.

Our study also compared the percentage of eyes where the IA absolute prediction error for the implanted IOL and preoperatively planned formula absolute prediction error was $\leq 0.50 \mathrm{D}$. In this analysis, the percentage of eyes with an absolute prediction error $\leq 0.50 \mathrm{D}$ was significantly higher with IA $(83.4 \%)$ than with the preoperative formula planned IOL $(76.5 \% ; p<0.0001)$. This difference was even larger for eyes where the preoperatively planned spherical lens power was different from the spherical power of the SN6AT3 IOL (83.3\% versus $68.8 \%$ respectively, $p<0.0001$ ).

These findings are consistent with data from a smaller study in eyes with prior myopic LASIK surgery, which demonstrated that IA is significantly more accurate than other methods in calculating IOL power. ${ }^{4}$ The use of IA resulted in a significantly lower mean absolute value of the prediction error than the other methods (all $p<0.0001$ ), with $67 \%$ of eyes analyzed with the IA system being within 0.50 D. In contrast, authors of another study ( $\mathrm{n}=295$ eyes) concluded that IA was only comparable to, but not superior to, conventional biometry with the IOLMaster ${ }^{\circledR}$ (Carl Zeiss Meditec AG, Jena, Germany) and surgeon's best choice. However, interpretation of these results may be limited by the lack of consistency in type of lens implanted, and the comparatively small sample size. ${ }^{12}$

Although IA does not replace surgeon's judgment, it does inform that judgment. In a retrospective study of 246 eyes, the IA-recommended lens power agreed with the preoperatively planned lens for $13 \%$ of eyes. ${ }^{4}$ In $30 \%$ of eyes, the 2 possible lens powers differed, and the IArecommended lens was chosen. In another $38 \%$ where they also differed, neither the IA-recommended nor the preoperatively planned lens was chosen, suggesting that the IA recommendation influenced the surgeon's decision. ${ }^{4}$ Similarly, in these analyses, when the powers of the IArecommended lens and the preoperatively planned lens differed, the IA-recommended lens power was used more often than the preoperatively planned lens.

Published studies comparing preoperative measurement and IA for selection of intraocular lens sphere power have reported conflicting results. ${ }^{4-8}$ However, these studies were comparatively small, which may have played a role when lack of a statistical significance was reported. ${ }^{4-8}$ In contrast, based on statistical guidelines, ${ }^{10}$ our study was large enough to detect significant differences.

Toric IOLs have a clear refractive benefit over spherical IOLs in eyes with low corneal astigmatism. ${ }^{4}$ In general, corneal preoperative astigmatism treated in our study was low (mean \pm SD: $1.22 \pm 0.44$ ). Thus, these results are meaningful for the clinical setting where minor corneal astigmatism is common among people presenting for cataract surgery, with up to $78 \%$ of patients presenting with astigmatism of $0.25-1.50 \mathrm{D}^{13-15} \mathrm{~A}$ further strength of the study is its use of real world data, which provides insight into results obtained during typical surgical practice rather than under the controlled circumstances of a clinical trial.

The main limitation of this study was the inability to compare the IA-predicted magnitude of postoperative refractive astigmatism calculated for the SN6AT3 IOL with the predicted astigmatism for the preoperatively planned lens, because neither value can be captured in the database. Moreover, the database does not currently capture the IArecommended cylinder power or data needed to calculate potential errors due to differences between the preoperatively planned axis and the implanted axis. The analysis focused on 
implantation of only one low-power toric IOL model. In addition, surgeons could have used any of several formulae to make a preoperative choice of lens. These analyses group together data from all formulae. Only a few formulae had sufficient data for comparison, and these provided comparable results. We cannot determine whether newer formulae provide notable improvement compared with older formulae. Moreover, these analyses examine only one model of lowpower toric intraocular lenses from a single manufacturer. In a previous study reporting on outcomes across the Alcon Acrysof platform, the Acrysof toric lenses were examined in a sub-analysis. The toric group was composed of lenses with cylinder powers ranging from $1.50 \mathrm{D}$ (T3) through T9. The absolute prediction error results were similar to those reported in this study. However, the mean paired differences were not analyzed in the sub-analysis.

\section{Conclusion}

The results of this retrospective analysis of patients who received SN6AT3 IOL implants demonstrated that IA produces more accurate spherical equivalent outcomes than the formula used preoperatively by the surgeon.

\section{Acknowledgments}

Editorial support was provided by Angela Cimmino, PharmD, and Jennie G. Jacobson, PhD, CMPP, of Fishawack Communications, Conshohocken, PA.

\section{Author contributions}

All authors contributed to the concept and design of the study, data analysis and interpretation, and critical revision of the manuscript. Dr. Cionni was in charge of data acquisition. All authors gave final approval of the version to be published, and agree to be accountable for all aspects of the work.

\section{Disclosure}

Drs Breen, Hamilton, and Williams are employees of Alcon Laboratories. Dr Cionni serves as a consultant to Alcon Laboratories, Morcher $\mathrm{GmbH}$, Glaukos, Omeros, Johnson \& Johnson, and receives royalties from Morcher $\mathrm{GmbH}$. Dr Cionni also reports personal fees from Alcon Laboratories, during the conduct of the study. The authors report no other conflicts of interest in this work.

\section{References}

1. Olsen T. Calculation of intraocular lens power: a review. Acta Ophthalmol Scand. 2007;85(5):472-485. doi:10.1111/j.17553768.2007.00879.x

2. Lee H, Kim TI, Kim EK. Corneal astigmatism analysis for toric intraocular lens implantation: precise measurements for perfect correction. Curr Opin Ophthalmol. 2015;26(1):34-38. doi:10.1097/ ICU.0000000000000119

3. Elhofi AH, Helaly HA. Comparison between digital and manual marking for toric intraocular lenses: a randomized trial. Medicine (Baltimore). 2015;94(38):e1618. doi:10.1097/MD.0000000000000874

4. Ianchulev T, Hoffer KJ, Yoo SH, et al. Intraoperative refractive biometry for predicting intraocular lens power calculation after prior myopic refractive surgery. Ophthalmology. 2014;121(1):56-60. doi:10.1016/j.ophtha.2013.08.041

5. Woodcock MG, Lehmann R, Cionni RJ, Breen M, Scott MC. Intraoperative aberrometry versus standard preoperative biometry and a toric IOL calculator for bilateral toric IOL implantation with a femtosecond laser: one-month results. J Cataract Refract Surg. 2016;42(6):817-825. doi:10.1016/j.jcrs.2016.02.048

6. Davison JA, Potvin R. Preoperative measurement vs intraoperative aberrometry for the selection of intraocular lens sphere power in normal eyes. Clin Ophthalmol. 2017;11:923-929. doi:10.2147/ OPTH.S135659

7. Solomon KD, Sandoval HP, Ptovin R. Correcting astigmatism at the time of cataract surgery: toric intraocular lenses and corneal relaxing incisions planned with an image-guidance system and intraoperative aberrometer versus manual planning and surgery. $J$ Cataract Refract Surg. 2019. Accessed April 16, 2019. doi:10.1016/j.jcrs.2018.12.002

8. Sudhakar S, Hill DC, King TS, et al. Intraoperative aberrometry versus preoperative biometry for intraocular lens power selection in short eyes. J Cataract Refract Surg. 2019. Accessed April, 2019. doi:10.1016/j.jcrs.2018.12.016

9. AcrySof ${ }^{\circledR} I Q$ Toric IOL [Product Information]. Fort Worth, TX: Alcon Laboratories, Inc.; 2014. Available from: https://www.myal con.com/products/surgical/acrysof-iq-toric-iol/specifications.shtml. Accessed March 18, 2019.

10. Harrell FE. Regression Modeling Strategies: With Applications to Linear Models, Logistic Regression, and Survival Analysis. New York: Springer Verlag; 2000:61.

11. Cionni RJ, Dimalanta R, Breen M, Hamilton C. A large retrospective database analysis comparing outcomes of intraoperative aberrometry with conventional preoperative planning. J Cataract Refract Surg. 2018;44:1230-1235. doi:10.1016/j.jcrs.2018.07.016

12. Zhang Z, Thomas LW, Leu SY, Carter S, Garg S. Refractive outcomes of intraoperative wavefront aberrometry versus optical biometry alone for intraocular lens power calculation. Indian J Ophthalmol. 2017;65(9):813-817. doi:10.4103/ijo.IJO_163_17

13. Curragh DS, Hassett P. Prevalence of corneal astigmatism in an NHS cataract surgery practice in Northern Ireland. Ulster Med J. 2017;86 (1):25-27.

14. Khan MI, Muhtaseb M. Prevalence of corneal astigmatism in patients having routine cataract surgery at a teaching hospital in the United Kingdom. $J$ Cataract Refract Surg. 2011;37(10):1751-1755. doi:10.1016/j.jcrs.2011.04.026

15. Ferrer-Blasco T, Montés-Micó R, Peixoto-de-Matos SC, GonzálezMéijome JM, Cerviño A. Prevalence of corneal astigmatism before cataract surgery. $J$ Cataract Refract Surg. 2009;35(1):70-75. doi:10.1016/j.jcrs.2008.09.027 


\section{Publish your work in this journal}

Clinical Ophthalmology is an international, peer-reviewed journal covering all subspecialties within ophthalmology. Key topics include: Optometry; Visual science; Pharmacology and drug therapy in eye diseases; Basic Sciences; Primary and Secondary eye care; Patient Safety and Quality of Care Improvements. This journal is indexed on PubMed
Central and CAS, and is the official journal of The Society of Clinical Ophthalmology (SCO). The manuscript management system is completely online and includes a very quick and fair peer-review system, which is all easy to use. Visit http://www.dovepress.com/ testimonials.php to read real quotes from published authors. 